\title{
On the generalized Hyers-Ulam-Rassias stability problem of radical functional equations
}

\author{
Seong Sik Kim', Yeol Je Cho ${ }^{2 *}$ and Madjid Eshaghi Gordjij,
}

\footnotetext{
"Correspondence: yjcho@gnu.ac.kr

${ }^{2}$ Department of Mathematics

Education and the RINS,

Gyeongsang National University,

Chinju, 660-701, Korea

Full list of author information is

available at the end of the article
}

\begin{abstract}
In this paper, the generalized Hyers-Ulam-Rassias stability problem of radical quadratic and radical quartic functional equations in quasi- $\beta$-Banach spaces and then the stability by using subadditive and subquadratic functions for radical functional equations in $(\beta, p)$-Banach spaces are given.

MSC: 39B82; 39B52; 46H 25

Keywords: radical functional equations; Hyers-Ulam-Rassias stability; quasi- $\beta$-normed spaces; subadditive and subquadratic functions
\end{abstract}

\section{Introduction}

In 1960, the stability problem of functional equations originated from the question of Ulam [1, 2] concerning the stability of group homomorphisms. The famous Ulam stability problem was partially solved by Hyers [3] in Banach spaces. Later, Aoki [4] and Bourgin [5] considered the stability problem with unbounded Cauchy differences. Rassias [6-9] provided a generalization of Hyers' theorem by proving the existence of unique linear mappings near approximate additive mappings. On the other hand, Rassias [10, 11] considered the Cauchy difference controlled by a product of different powers of norm. The above results have been generalized by Forti [12] and Gǎvruta [13], who permitted the Cauchy difference to become arbitrary unbounded. Gajda and Ger [14] showed that one can get analogous stability results for subadditive multifunctions. Gruber [15] remarked that Ulam's problem is of particular interest in probability theory and in the case of functional equations of different types. Recently, Baktash et al. [16], Cho et al. [17-20], Gordji et al. [21-24], Lee et al. [25, 26], Najati et al. [27, 28], Park et al. [29], Saadati et al. [30] and Savadkouhi et al. [31] have studied and generalized several stability problems of a large variety of functional equations.

The most famous functional equation is the Cauchy equation

$$
f(x+y)=f(x)+f(y),
$$

any solution of which is called additive. It is easy to see that the function $f: \mathbb{R} \rightarrow \mathbb{R}$ defined by $f(x)=c x^{2}$, where $c$ is an arbitrary constant, is a solution of the functional equation

$$
f(x+y)+f(x-y)=2 f(x)+2 f(y) .
$$

@ 2012 Kim et al.; licensee Springer. This is an Open Access article distributed under the terms of the Creative Commons Attribution License (http://creativecommons.org/licenses/by/2.0), which permits unrestricted use, distribution, and reproduction in any medium, provided the original work is properly cited. 
Thus it is natural that each equation is said to be a quadratic functional equation. In particular, every solution of the quadratic equation (1.1) is said to be a quadratic function.

Lee et al. [32] considered the following functional equation:

$$
f(2 x+y)+f(2 x-y)=4 f(x+y)+4 f(x-y)+24 f(x)-6 f(y) .
$$

The functional equation (1.2) clearly has $f(x)=c x^{4}$ as a solution when $f$ is a real valued function of a real variable. So, it is said to be a quartic functional equation.

Before we present our results, we introduce here some basic facts concerning quasi- $\beta$ normed space and preliminary results. Let $\beta$ be a real number with $0<\beta \leq 1$, and $\mathbb{K}$ be either $\mathbb{R}$ or $\mathbb{C}$. Let $X$ be a linear space over $\mathbb{C}$. A quasi- $\beta$-norm $\|\cdot\|$ is a real-valued function on $X$ satisfying the following:

(1) $\|x\| \geq 0$ for all $x \in X$ and $\|x\|=0$ if and only if $x=0$;

(2) $\|\lambda x\|=|\lambda|^{\beta} \cdot\|x\|$ for all $\lambda \in \mathbb{R}$ and $x \in X$;

(3) There is a constant $K \geq 1$ such that $\|x+y\| \leq K(\|x\|+\|y\|)$ for all $x, y \in X$.

Then $(X,\|\cdot\|)$ is called a quasi- $\beta$-normed space if $\|\cdot\|$ is a quasi- $\beta$-norm on $X$. The smallest possible $K$ is called the module of concavity of $\|\cdot\|$. A quasi- $\beta$-Banach space ia a complete quasi- $\beta$-normed space.

A quasi- $\beta$-norm $\|\cdot\|$ is called a $(\beta, p)$-norm $(0<p \leq 1)$ if $\|x+y\|^{p} \leq\|x\|^{p}+\|y\|^{p}$ for all $x, y \in X$. In this case, a quasi- $\beta$-Banach space is called a $(\beta, p)$-Banach space.

In [33], Tabor investigated a version of the Hyers-Rassias-Gajda theorem in quasiBanach spaces. For further details on quasi- $\beta$ normed spaces and $(\beta, p)$-Banach space, we refer to the papers [34-37].

In this paper, we consider the following functional equations:

$$
\begin{aligned}
& f\left(\sqrt{x^{2}+y^{2}}\right)=f(x)+f(y), \\
& f\left(\sqrt{x^{2}+y^{2}}\right)+f\left(\sqrt{\left|x^{2}-y^{2}\right|}\right)=2 f(x)+2 f(y),
\end{aligned}
$$

and discuss the generalized Hyers-Ulam-Rassias stability problem in quasi- $\beta$-normed spaces and then the stability by using subadditive and subquadratic functions for the functional equations (1.3) and (1.4) in $(\beta, p)$-Banach spaces.

\section{Stability of radical functional equations}

Using an idea of Găvruta [13], we prove the generalized stability of (1.3) and (1.4) in the spirit of Ulam, Hyers and Rassias.

In [38], Khodaei et al. proved the following result:

Lemma 2.1 Let $X$ be a real linear space and $f: \mathbb{R} \rightarrow X$ be a function. Then we have the following:

(1) Iff satisfies the functional equation (1.3), then $f$ is quadratic.

(2) Iff satisfies the functional equation (1.4), then $f$ is quartic.

Proof We will only prove (2). Letting $x=y=0$ in (1.4), we get $f(0)=0$. Setting $x=-x$ in (1.4), we have

$$
f\left(\sqrt{x^{2}+y^{2}}\right)+f\left(\sqrt{\left|x^{2}-y^{2}\right|}\right)=2 f(-x)+2 f(y)
$$


for all $x, y \in \mathbb{R}$. If we compare (1.4) with (2.1), we obtain that $f(-x)=f(x)$ for all $x \in \mathbb{R}$. Letting $y=x$ in (1.4) and then using $f(0)=0$ and the evenness of $f$, we have $f(\sqrt{2} x)=4 f(x)$ for all $x \in \mathbb{R}$. Putting $y=\sqrt{2} x$ in (1.4) and using $f(\sqrt{2} x)=4 f(x)$, we get $f(\sqrt{3} x)=9 f(x)$ for all $x \in \mathbb{R}$. By induction, we lead to $f(\sqrt{n} x)=n^{2} f(x)$ for all $x \in \mathbb{R}$ and $n \in \mathbb{Z}^{+}$. We obtain $f\left(\frac{x}{\sqrt{n}}\right)=\frac{1}{n^{2}} f(x)$, and so $f\left(\sqrt{\frac{m}{n}} x\right)=\frac{m^{2}}{n^{2}} f(x)$ for all $x \in \mathbb{R}$ and $m, n \in \mathbb{Z}^{+}$. So, we have

$$
f(\sqrt{r} x)=r^{2} f(x)
$$

for all $x \in \mathbb{R}$ and all $r \in \mathbb{Q}^{+}$. Replacing $x$ and $y$ by $x+y$ and $x-y$ in (1.4) respectively, we obtain

$$
f\left(\sqrt{2 x^{2}+2 y^{2}}\right)+f(\sqrt{|4 x y|})=2 f(x+y)+2 f(x-y)
$$

for all $x, y \in \mathbb{R}$. Setting $y=\sqrt{x^{2}+y^{2}}$ in (1.4) and using the evenness of $f$, we get

$$
f\left(\sqrt{2 x^{2}+y^{2}}\right)-2 f\left(\sqrt{x^{2}+y^{2}}\right)=2 f(x)-f(y)
$$

for all $x, y \in \mathbb{R}$. Putting $x=2 x$ in (2.3) and using (2.2), we get

$$
2 f\left(\sqrt{4 x^{2}+y^{2}}\right)+32 f(\sqrt{|x y|})=f(2 x+y)+f(2 x-y)
$$

for all $x, y \in \mathbb{R}$. Setting $x=\sqrt{2} x$ in (1.4) and using (2.2), we get

$$
f\left(\sqrt{4 x^{2}+y^{2}}\right)-2 f\left(\sqrt{2 x^{2}+y^{2}}\right)=8 f(x)-f(y)
$$

for all $x, y \in \mathbb{R}$. It follows from (2.2), (2.3), (2.4) and (2.6) that

$$
f\left(\sqrt{4 x^{2}+y^{2}}\right)+16 f(\sqrt{|x y|})=2 f(x+y)+2 f(x-y)+12 f(x)-3 f(y)
$$

for all $x, y \in \mathbb{R}$. So it follows from (2.5) and (2.7) that $f$ satisfies (1.2). Therefore, $f$ is quartic. This completes the proof.

Let $\mathcal{X}$ be a quasi- $\beta$-Banach space with the quasi- $\beta$-norm $\|\cdot\|_{\mathcal{X}}$ and $K$ be the modulus of concavity of $\|\cdot\|_{\mathcal{X}}$. Let $\phi, \psi: \mathbb{R}^{2} \rightarrow \mathbb{R}^{+} \cup\{0\}$ be functions. A function $f: \mathbb{R} \rightarrow \mathcal{X}$ is said to be $\phi$-approximatively radical quadratic if

$$
\left\|f\left(\sqrt{x^{2}+y^{2}}\right)-f(x)-f(y)\right\|_{\mathcal{X}} \leq \phi(x, y)
$$

and a function $f: \mathbb{R} \rightarrow \mathcal{X}$ is said to be $\psi$-approximatively radical quartic if

$$
\left\|f\left(\sqrt{x^{2}+y^{2}}\right)+f\left(\sqrt{\left|x^{2}-y^{2}\right|}\right)-2 f(x)-2 f(y)\right\|_{\mathcal{X}} \leq \psi(x, y)
$$

for all $x, y \in \mathbb{R}$. 
Theorem 2.2 Let $f: \mathbb{R} \rightarrow \mathcal{X}$ be a $\phi$-approximatively radical quadratic function with $f(0)=0$. If a function $\phi: \mathbb{R}^{2} \rightarrow \mathbb{R}^{+} \cup\{0\}$ satisfies

$$
\widehat{\phi}(x):=\sum_{j=0}^{\infty}\left(\frac{K}{2^{\beta}}\right)^{j}\left(\phi\left(2^{\frac{j}{2}} x, 2^{\frac{j}{2}} x\right)+\phi\left(2^{\frac{j+1}{2}} x, 0\right)\right)<\infty
$$

and $\lim _{n \rightarrow \infty} \frac{1}{2^{\beta n}} \phi\left(2^{\frac{n}{2}} x, 2^{\frac{n}{2}} y\right)=0$ for all $x, y \in \mathbb{R}$, then the limit $\mathcal{F}(x):=\lim _{n \rightarrow \infty} \frac{1}{2^{n}} f\left(2^{\frac{n}{2}} x\right)$ exists for all $x \in \mathbb{R}$ and a function $\mathcal{F}: \mathbb{R} \rightarrow \mathcal{X}$ is unique quadratic satisfying the functional equation (1.3) and the inequality

$$
\|f(x)-\mathcal{F}(x)\|_{\mathcal{X}} \leq \frac{K}{2^{\beta}} \widehat{\phi}(x)
$$

for all $x \in \mathbb{R}$

Proof Replacing $x$ and $y$ by $\frac{x+y}{\sqrt{2}}$ and $\frac{x-y}{\sqrt{2}}$ in (2.8) respectively, we get

$$
\left\|f\left(\sqrt{x^{2}+y^{2}}\right)-f\left(\frac{x+y}{\sqrt{2}}\right)-f\left(\frac{x-y}{\sqrt{2}}\right)\right\|_{\mathcal{X}} \leq \phi\left(\frac{x+y}{\sqrt{2}}, \frac{x-y}{\sqrt{2}}\right)
$$

for all $x, y \in \mathbb{R}$. It follows from (2.8) and (2.11) that

$$
\left\|f(x)+f(y)-f\left(\frac{x+y}{\sqrt{2}}\right)-f\left(\frac{x-y}{\sqrt{2}}\right)\right\|_{\mathcal{X}} \leq K\left(\phi(x, y)+\phi\left(\frac{x+y}{\sqrt{2}}, \frac{x-y}{\sqrt{2}}\right)\right)
$$

for all $x, y \in \mathbb{R}$. Setting $y=x$ in (2.12), it follows from $f(0)=0$ that

$$
\|2 f(x)-f(\sqrt{2} x)\|_{\mathcal{X}} \leq K(\phi(x, x)+\phi(\sqrt{2} x, 0))
$$

and so

$$
\left\|f(x)-\frac{1}{2} f(\sqrt{2} x)\right\|_{\mathcal{X}} \leq \frac{K}{2^{\beta}}(\phi(x, x)+\phi(\sqrt{2} x, 0))
$$

for all $x \in \mathbb{R}$. For any integers $m, k$ with $m>k \geq 0$,

$$
\left\|\frac{1}{2^{k}} f\left(2^{\frac{k}{2}} x\right)-\frac{1}{2^{m}} f\left(2^{\frac{m}{2}} x\right)\right\|_{\mathcal{X}} \leq \frac{K}{2^{\beta}} \sum_{j=k}^{m-1}\left(\frac{K}{2^{\beta}}\right)^{j}\left(\phi\left(2^{\frac{j}{2}} x, 2^{\frac{j}{2}} x\right)+\phi\left(2^{\frac{j+1}{2}} x, 0\right)\right)
$$

for all $x \in \mathbb{R}$. Then a sequence $\left\{\frac{1}{2^{n}} f\left(2^{\frac{n}{2}} x\right)\right\}$ is a Cauchy sequence in a quasi- $\beta$-Banach space $\mathcal{X}$ and so it converges. We can define a function $\mathcal{F}: \mathbb{R} \rightarrow \mathcal{X}$ by $\mathcal{F}(x):=\lim _{n \rightarrow \infty} \frac{1}{2^{n}} f\left(2^{\frac{n}{2}} x\right)$ for all $x \in \mathbb{R}$. From (2.8), the following inequality holds:

$$
\begin{aligned}
& \left\|\mathcal{F}\left(\sqrt{x^{2}+y^{2}}\right)-\mathcal{F}(x)-\mathcal{F}(y)\right\|_{\mathcal{X}} \\
& \quad=\lim _{n \rightarrow \infty} \frac{1}{2^{\beta n}}\left\|f\left(\sqrt{2^{n} x^{2}+2^{n} y^{2}}\right)-f\left(2^{\frac{n}{2}} x\right)-f\left(2^{\frac{n}{2}} y\right)\right\|_{\mathcal{X}} \\
& \leq \lim _{n \rightarrow \infty} \frac{1}{2^{\beta n}} \phi\left(2^{\frac{n}{2}} x, 2^{\frac{n}{2}} y\right)=0
\end{aligned}
$$


for all $x, y \in \mathbb{R}$. Then $\mathcal{F}\left(\sqrt{x^{2}+y^{2}}\right)-\mathcal{F}(x)-\mathcal{F}(y)=0$ and, by Lemma 2.1, $\mathcal{F}$ is a quadratic function. Taking the limit $m \rightarrow \infty$ in (2.15) with $k=0$, we find that a function $\mathcal{F}$ satisfies (2.10) near the approximate function $f$ of the functional equation (1.3).

Next, we assume that there exists another quadratic function $\mathcal{G}: \mathbb{R} \rightarrow \mathcal{X}$ which satisfies the functional equation (1.3) and (2.10). Since $\mathcal{G}$ satisfies (1.3), it easy to show that $\mathcal{G}\left(2^{\frac{n}{2}} x\right)=$ $2^{n} \mathcal{G}(x)$ for all $x \in \mathbb{R}$ and $n \geq 1$. Then we have

$$
\begin{aligned}
\|\mathcal{F}(x)-\mathcal{G}(x)\| & =\left\|\frac{1}{2^{n}} \mathcal{F}\left(2^{\frac{n}{2}} x\right)-\frac{1}{2^{n}} \mathcal{G}\left(2^{\frac{n}{2}} x\right)\right\| \\
& \leq \frac{K}{2^{\beta n}}\left(\left\|\mathcal{F}\left(2^{\frac{n}{2}} x\right)-f\left(2^{\frac{n}{2}} x\right)\right\|+\left\|f\left(2^{\frac{n}{2}} x\right)-\mathcal{G}\left(2^{\frac{n}{2}} x\right)\right\|\right) \\
& \leq \frac{K^{2-n}}{2^{\beta-1}} \sum_{k=n}^{\infty}\left(\frac{K}{2^{\beta}}\right)^{k}\left(\phi\left(2^{\frac{k}{2}} x, 2^{\frac{k}{2}} x\right)+\phi\left(2^{\frac{k+1}{2}} x, 0\right)\right)
\end{aligned}
$$

for all $x \in \mathbb{R}$. Letting $n \rightarrow \infty$, we establish $\mathcal{F}(x)=\mathcal{G}(x)$ for all $x \in \mathbb{R}$. This completes the proof.

Theorem 2.3 Let $f: \mathbb{R} \rightarrow \mathcal{X}$ be a $\phi$-approximatively radical quadratic function. If a function $\phi: \mathbb{R}^{2} \rightarrow \mathbb{R}^{+} \cup\{0\}$ satisfies

$$
\widehat{\phi}(x):=\sum_{j=1}^{\infty}\left(K 2^{\beta}\right)^{j}\left(\phi\left(2^{-\frac{j}{2}} x, 2^{-\frac{j}{2}} x\right)+\phi\left(2^{\frac{1-j}{2}} x, 0\right)\right)<\infty
$$

and $\lim _{n \rightarrow \infty} 2^{\beta n} \phi\left(2^{-\frac{n}{2}} x, 2^{-\frac{n}{2}} y\right)=0$ for all $x, y \in \mathbb{R}$, then the limit $\mathcal{F}(x):=\lim _{n \rightarrow \infty} 2^{n} f\left(2^{-\frac{n}{2}} x\right)$ exists for all $x \in \mathbb{R}$ and a function $\mathcal{F}: \mathbb{R} \rightarrow \mathcal{X}$ is unique quadratic satisfying the functional equation (1.3) and the inequality

$$
\|f(x)-\mathcal{F}(x)\|_{\mathcal{X}} \leq \frac{K}{2^{\beta}} \widehat{\phi}(x)
$$

for all $x \in \mathbb{R}$

Proof If $x$ is replaced by $2^{-\frac{1}{2}} x$ in the inequality (2.13), then the proof follows from the proof of Theorem 2.2.

Corollary 2.4 Let $r, s \in \mathbb{R}^{+} \cup\{0\}$ and $\varepsilon \geq 0$. If a function $f: \mathbb{R} \rightarrow \mathcal{X}$ satisfies the following inequality:

$$
\left\|f\left(\sqrt{x^{2}+y^{2}}\right)-f(x)-f(y)\right\|_{\mathcal{X}} \leq\left\{\begin{array}{l}
\varepsilon ; \\
\varepsilon|x|^{r}|y|^{s}
\end{array}\right.
$$

for all $x, y \in \mathbb{R}$, then there exists a unique quadratic function $\mathcal{F}: \mathbb{R} \rightarrow \mathcal{X}$ satisfying the functional equation (1.3) and the following inequality:

$$
\|f(x)-\mathcal{F}(x)\|_{\mathcal{X}} \leq \begin{cases}\frac{2 \varepsilon K}{2^{\beta}-K}, & K<2^{\beta} ; \\ \frac{\varepsilon K|x|^{r+s}}{2^{\beta}-K \sqrt{2^{r+s}}}, & r+s<2 \beta-2 \log _{2} K\end{cases}
$$

for all $x \in \mathbb{R}$. 
Corollary 2.5 Let $r, s \in \mathbb{R}^{+} \cup\{0\}$ and $\varepsilon \geq 0$. If a function $f: \mathbb{R} \rightarrow \mathcal{X}$ satisfies the following inequality:

$$
\left\|f\left(\sqrt{x^{2}+y^{2}}\right)-f(x)-f(y)\right\|_{\mathcal{X}} \leq \varepsilon\left(|x|^{r}+|y|^{s}\right)
$$

for all $x, y \in \mathbb{R}$, then there exists a unique quadratic function $\mathcal{F}: \mathbb{R} \rightarrow \mathcal{X}$ satisfying the functional equation (1.3) and the following inequality:

$$
\|f(x)-\mathcal{F}(x)\|_{\mathcal{X}} \leq \varepsilon K\left(\frac{\left(1+\sqrt{2^{r}}\right)|x|^{r}}{2^{\beta}-K \sqrt{2^{r}}}+\frac{|x|^{s}}{2^{\beta}-K \sqrt{2^{s}}}\right), \quad r, s<2 \beta-2 \log _{2} K
$$

for all $x \in \mathbb{R}$.

Now, we give an example to illustrate that the functional equation (1.3) is not stable for $r=2=s$ in a quasi-1-Banach space with $K=1$.

Example 2.6 Let $\phi: \mathbb{R} \rightarrow \mathbb{R}$ be defined by

$$
\phi(x):= \begin{cases}x^{2} & \text { for }|x|<1 \\ 1 & \text { for }|x| \geq 1\end{cases}
$$

Consider the function $f: \mathbb{R} \rightarrow \mathbb{R}$ by the formula

$$
f(x):=\sum_{m=0}^{\infty} \frac{1}{4^{m}} \phi\left(2^{m} x\right)
$$

for all $x \in \mathbb{R}$. It is clear that $f$ is bounded by $\frac{4}{3}$ on $\mathbb{R}$. We prove that

$$
\left|f\left(\sqrt{x^{2}+y^{2}}\right)-f(x)-f(y)\right| \leq 16\left(|x|^{2}+|y|^{2}\right)
$$

for all $x, y \in \mathbb{R}$. To see this, if $|x|^{2}+|y|^{2}=0$ or $|x|^{2}+|y|^{2} \geq \frac{1}{4}$, then we have (2.16). Now suppose that $0<|x|^{2}+|y|^{2}<\frac{1}{4}$. Then there exists a positive integer $k$ such that

$$
\frac{1}{4^{k+1}}<|x|^{2}+|y|^{2}<\frac{1}{4^{k}}
$$

so that $2^{m}|x|, 2^{m}|y|, 2^{m} \sqrt{x^{2}+y^{2}} \in(-1,1)$ for all $m=0,1, \ldots, k-1$. It follows from the definition of $f$ and (2.17) that

$$
\begin{aligned}
& \left|f\left(\sqrt{x^{2}+y^{2}}\right)-f(x)-f(y)\right| \\
& \quad \leq \sum_{j=k}^{\infty} \frac{1}{4^{j}}\left|\phi\left(2^{j} \sqrt{x^{2}+y^{2}}\right)-\phi\left(2^{j} x\right)-\phi\left(2^{j} y\right)\right| \\
& \quad \leq 3 \sum_{j=k}^{\infty} \frac{1}{4^{j}}=\frac{16}{4^{k+1}} \leq 16\left(|x|^{2}+|y|^{2}\right)
\end{aligned}
$$

for all $x, y \in \mathbb{R}$ with $|x|^{2}+|y|^{2}<\frac{1}{4}$. Thus, the condition (2.16) holds true. 
Next, we claim that the quadratic equation (1.3) is not stable for $r=2=s$. Assume that there exist a quadratic function $\mathcal{F}: \mathbb{R} \rightarrow \mathbb{R}$ and a positive constant $\mu$ such that $\mid f(x)-$ $\left.\mathcal{F}(x)|\leq \mu| x\right|^{2}$ for all $x \in \mathbb{R}$. Then there exists a constant $c \in \mathbb{R}$ such that $\mathcal{F}(x)=c x^{2}$ for all $x \in \mathbb{R}$. So we have

$$
|f(x)| \leq(\mu+|c|)|x|^{2}
$$

for all $x \in \mathbb{R}$. But, we can choose a $\lambda \in \mathbb{Z}^{+}$with $\lambda>\mu+|c|$. If $x \in\left(0,2^{-\lambda}\right)$, then $2^{j} x \in(0,1)$ for all $j=0,1, \ldots, \lambda-1$. For this $x$, we obtain

$$
|f(x)|=\left|\sum_{j=0}^{\infty} \frac{\phi\left(2^{j} x\right)}{4^{j}}\right| \geq \sum_{j=0}^{\lambda-1} \frac{\phi\left(2^{j} x\right)}{4^{j}}=\lambda x^{2}>(\mu+|c|)|x|^{2},
$$

which contradicts (2.18). Therefore, the quadratic equation (1.3) is not stable for $r=2=s$ in Corollary 2.5.

Theorem 2.7 Let $f: \mathbb{R} \rightarrow \mathcal{X}$ be a $\psi$-approximatively radical quartic function with $f(0)=$ 0 . If the function $\psi: \mathbb{R}^{2} \rightarrow \mathbb{R}^{+} \cup\{0\}$ satisfies

$$
\widehat{\psi}(x):=\sum_{j=0}^{\infty}\left(\frac{K}{4^{\beta}}\right)^{j}\left(\psi\left(2^{\frac{j}{2}} x, 2^{\frac{j}{2}} x\right)+\frac{1}{2} \psi\left(2^{\frac{j+1}{2}} x, 0\right)\right)<\infty
$$

and $\lim _{n \rightarrow \infty} \frac{1}{4^{\beta n}} \psi\left(2^{\frac{n}{2}} x, 2^{\frac{n}{2}} y\right)=0$ for all $x, y \in \mathbb{R}$, then the limit $\mathcal{H}(x):=\lim _{n \rightarrow \infty} \frac{1}{4^{n}} f\left(2^{\frac{n}{2}} x\right)$ for all $x \in \mathbb{R}$ exists and a function $\mathcal{H}: \mathbb{R} \rightarrow \mathcal{X}$ is unique quartic satisfying the functional equation (1.4) and the inequality

$$
\|f(x)-\mathcal{H}(x)\|_{\mathcal{X}} \leq \frac{K^{2}}{2^{3 \beta-1}} \widehat{\psi}(x)
$$

for all $x \in \mathbb{R}$.

Proof Replacing $x$ and $y$ by $\sqrt{2} x$ and $\sqrt{2} y$ in (2.9) respectively, we have

$$
\left\|f\left(\sqrt{2 x^{2}+2 y^{2}}\right)+f\left(\sqrt{\left|2 x^{2}-2 y^{2}\right|}\right)-2 f(\sqrt{2} x)-2 f(\sqrt{2} y)\right\|_{\mathcal{X}} \leq \psi(\sqrt{2} x, \sqrt{2} y)
$$

for all $x, y \in \mathbb{R}$. Again, replacing $x$ and $y$ by $x+y$ and $x-y$ in (2.9) respectively, we get

$$
\left\|f\left(\sqrt{2 x^{2}+2 y^{2}}\right)+f(\sqrt{|4 x y|})-2 f(x+y)-2 f(x-y)\right\|_{\mathcal{X}} \leq \psi(x+y, x-y)
$$

for all $x, y \in \mathbb{R}$. It follows from (2.20) and (2.21) that

$$
\begin{aligned}
& \left\|2 f(\sqrt{2} x)+2 f(\sqrt{2} y)+f(\sqrt{|4 x y|})-f\left(\sqrt{\left|2 x^{2}-2 y^{2}\right|}\right)-2 f(x+y)-2 f(x-y)\right\|_{\mathcal{X}} \\
& \quad \leq K(\psi(\sqrt{2} x, \sqrt{2} y)+\psi(x+y, x-y))
\end{aligned}
$$

for all $x, y \in \mathbb{R}$. Setting $y=0$ in (2.22) and $f(0)=0$, we have

$$
\left\|2 f(\sqrt{2} x)-f\left(\sqrt{2 x^{2}}\right)-4 f(x)\right\|_{\mathcal{X}} \leq K(\psi(x, x)+\psi(\sqrt{2} x, 0))
$$


for all $x \in \mathbb{R}$. Setting $y=x$ in (2.9), we have

$$
\left\|f\left(\sqrt{2 x^{2}}\right)-4 f(x)\right\|_{\mathcal{X}} \leq \psi(x, x)
$$

for all $x \in \mathbb{R}$. It follows from (2.23) and (2.24) that

$$
\|2 f(\sqrt{2} x)-8 f(x)\|_{\mathcal{X}} \leq K^{2}(2 \psi(x, x)+\psi(\sqrt{2} x, 0))
$$

for all $x \in \mathbb{R}$. Since $f(0)=0$, it follows from (2.24) and (2.25) that

$$
\left\|f(x)-\frac{1}{4} f(\sqrt{2} x)\right\|_{\mathcal{X}} \leq \frac{K^{2}}{2^{3 \beta-1}}\left(\psi(x, x)+\frac{1}{2} \psi(\sqrt{2} x, 0)\right)
$$

for all $x \in \mathbb{R}$. Hence we have

$$
\begin{aligned}
& \left\|\frac{1}{4^{k}} f\left(2^{\frac{k}{2}} x\right)-\frac{1}{4^{m}} f\left(2^{\frac{m}{2}} x\right)\right\|_{\mathcal{X}} \\
& \leq \frac{K^{2}}{2^{3 \beta-1}} \sum_{j=k}^{m-1}\left(\frac{K}{4^{\beta}}\right)^{j}\left(\psi\left(2^{\frac{j}{2}} x, 2^{\frac{j}{2}} x\right)+\frac{1}{2} \psi\left(2^{\frac{j+1}{2}} x, 0\right)\right)
\end{aligned}
$$

for all $x \in \mathbb{R}$ and $m>k \geq 0$. Then a sequence $\left\{\frac{1}{4^{n}} f\left(2^{\frac{n}{2}} x\right)\right\}$ is a Cauchy sequence in $\mathcal{X}$, and so it converges to a point in $\mathcal{X}$. We can define a function $\mathcal{H}: \mathbb{R} \rightarrow \mathcal{X}$ by $\mathcal{H}(x):=$ $\lim _{n \rightarrow \infty} \frac{1}{4^{n}} f\left(2^{\frac{n}{2}} x\right)$ for all $x \in \mathbb{R}$. From (2.9), the following inequality holds:

$$
\left\|\mathcal{H}\left(\sqrt{x^{2}+y^{2}}\right)+\mathcal{H}\left(\sqrt{\left|x^{2}-y^{2}\right|}\right)-2 \mathcal{H}(x)-2 \mathcal{H}(y)\right\|_{\mathcal{X}} \leq \lim _{n \rightarrow \infty} \frac{1}{4^{\beta n}} \psi\left(2^{\frac{n}{2}} x, 2^{\frac{n}{2}} y\right)=0
$$

for all $x, y \in \mathbb{R}$. Then, we have $\mathcal{H}\left(\sqrt{x^{2}+y^{2}}\right)+\mathcal{H}\left(\sqrt{\left|x^{2}-y^{2}\right|}\right)-2 \mathcal{H}(x)-2 \mathcal{H}(y)=0$, and by Lemma 2.1, $\mathcal{H}$ is quartic. Taking the limit $m \rightarrow \infty$ in (2.27) with $k=0$, we obtain that a function $\mathcal{H}$ satisfies (2.19) near the approximate function $f$ of the functional equation (1.4). The remaining assertion is similar to the corresponding part of Theorem 2.2. This completes the proof.

Corollary 2.8 If there exist $r, s, t \in \mathbb{R}^{+} \cup\{0\}$ and $\varepsilon \geq 0$; if a function $f: \mathbb{R} \rightarrow \mathcal{X}$ satisfies the inequality

$$
\left\|f\left(\sqrt{x^{2}+y^{2}}\right)+f\left(\sqrt{\left|x^{2}-y^{2}\right|}\right)-2 f(x)-2 f(y)\right\|_{\mathcal{X}} \leq\left\{\begin{array}{l}
\varepsilon \\
\varepsilon|x|^{r}|y|^{s} \\
\varepsilon\left(|x|^{r}+|y|^{s}\right)
\end{array}\right.
$$

for all $x, y \in \mathbb{R}$, then there exists a unique quartic function $\mathcal{H}: \mathbb{R} \rightarrow \mathcal{X}$ which satisfies (1.4) and the inequality

$$
\|f(x)-\mathcal{H}(x)\|_{\mathcal{X}} \leq \begin{cases}\frac{3 \varepsilon K^{2}}{2^{3 \beta}-2^{\beta} K}, & K<4^{\beta} ; \\ \frac{\varepsilon K^{2}|x|^{r+s}}{2^{2 \beta}-\sqrt{2^{r+s}}}, & r+s<4 \beta-2 \log _{2} K ; \\ \frac{\varepsilon K^{2}}{2^{\beta-1}}\left(\frac{2+\sqrt{2^{r-2}}|x|^{r}}{4^{\beta}-K \sqrt{2^{r}}}+\frac{|x|^{s}}{4^{\beta}-K \sqrt{2^{s}}}\right), & r, s<4 \beta-2 \log _{2} K\end{cases}
$$

for all $x \in \mathbb{R}$. 
We recall that a subadditive function is a function $\phi: A \rightarrow B$ having a domain $A$ and a codomain $(B, \leq)$ that are both closed under addition with the following property:

$$
\phi(x+y) \leq \phi(x)+\phi(y)
$$

for all $x, y \in A$. Also, a subquadratic function is a function $\psi: A \rightarrow B$ with $\psi(0)=0$ and the following property:

$$
\psi(x+y)+\psi(x-y) \leq 2 \psi(x)+2 \psi(y)
$$

for all $x, y \in A$.

Let $\ell \in\{-1,1\}$ be fixed. If there exists a constant $L$ with $0<L<1$ such that a function $\phi: A \rightarrow B$ satisfies

$$
\ell \phi(x+y) \leq \ell L^{\ell}(\phi(x)+\phi(y))
$$

for all $x, y \in A$, then we say that $\phi$ is contractively subadditive if $\ell=1$ and $\phi$ is expansively superadditive if $\ell=-1$. It follows by the last inequality that $\phi$ satisfies the following properties:

$$
\phi\left(2^{\ell} x\right) \leq 2^{\ell} L \phi(x), \quad \phi\left(2^{\ell k} x\right) \leq\left(2^{\ell} L\right)^{k} \phi(x)
$$

for all $x \in A$ and $k \geq 1$.

Similarly, if there exists a constant $L$ with $0<L<1$ such that a function $\psi: A \rightarrow B$ with $\psi(0)=0$ satisfies

$$
\ell \psi(x+y)+\ell \psi(x-y) \leq 2 \ell L^{\ell}(\psi(x)+\psi(y))
$$

for all $x, y \in A$, then we say that $\psi$ is contractively subquadratic if $\ell=1$ and $\psi$ is expansively superquadratic if $\ell=-1$. It follows from the last inequality that $\psi$ satisfies the following properties:

$$
\psi\left(2^{\ell} x\right) \leq 4^{\ell} L \psi(x), \quad \psi\left(2^{\ell k} x\right) \leq\left(4^{\ell} L\right)^{k} \psi(x)
$$

for all $x \in A$ and $k \geq 1$.

From now on, we establish the modified Hyers-Ulam-Rassias stability of the equations (1.3) and (1.4) in a $(\beta, p)$-Banach space $\mathcal{Y}$.

Theorem 2.9 Let $f: \mathbb{R} \rightarrow \mathcal{Y}$ be a $\phi$-approximatively radical quadratic function with $f(0)=0$. Assume that the function $\phi$ is contractively subadditive with a constant $L$ satisfying $2^{1-2 \beta} L<1$. Then there exists a unique quadratic function $\mathcal{F}: \mathbb{R} \rightarrow \mathcal{Y}$ which satisfies (1.3) and the inequality

$$
\|f(x)-\mathcal{F}(x)\|_{\mathcal{Y}} \leq \frac{\widehat{\Phi}(x)}{\sqrt[p]{4^{\beta p}-(2 L)^{p}}}
$$


for all $x \in \mathbb{R}$, where

$$
\widehat{\Phi}(x):=K^{2}\left(2^{\beta}(\phi(x, x)+\phi(\sqrt{2} x, 0))+\phi(\sqrt{2} x, \sqrt{2} x)+\phi(2 x, 0)\right) .
$$

Proof Using (2.13) in the proof of Theorem 2.2, we get

$$
\left\|f(x)-\frac{1}{4} f(2 x)\right\|_{\mathcal{Y}} \leq \frac{1}{4^{\beta}} \widehat{\Phi}(x)
$$

for all $x \in \mathbb{R}$. Thus it follows from (2.29) that

$$
\begin{aligned}
\left\|\frac{1}{4^{k}} f\left(2^{k} x\right)-\frac{1}{4^{m}} f\left(2^{m} x\right)\right\|_{\mathcal{Y}}^{p} & \leq \frac{1}{4^{\beta p}} \sum_{j=k}^{m-1}\left(\frac{1}{4^{\beta}}\right)^{j p}(2 L)^{j p} \widehat{\Phi}(x)^{p} \\
& \leq \frac{\widehat{\Phi}(x)^{p}}{4^{\beta p}} \sum_{j=k}^{m-1}\left(2^{1-2 \beta} L\right)^{j p}
\end{aligned}
$$

for all $x \in \mathbb{R}$ and integers $m>k \geq 0$. Then a sequence $\left\{\frac{1}{4^{n}} f\left(2^{n} x\right)\right\}$ is a Cauchy sequence in a $(\beta, p)$-Banach space $\mathcal{Y}^{p}$, and so we can define a function $\mathcal{F}: \mathbb{R} \rightarrow \mathcal{Y}$ by $\mathcal{F}(x):=$ $\lim _{n \rightarrow \infty} \frac{1}{4^{n}} f\left(2^{n} x\right)$ for all $x \in \mathbb{R}$. Then, we get

$$
\begin{aligned}
& \left\|\mathcal{F}\left(\sqrt{x^{2}+y^{2}}\right)-\mathcal{F}(x)-\mathcal{F}(y)\right\|_{\mathcal{Y}}^{p} \\
& \quad \leq \lim _{n \rightarrow \infty} \frac{1}{4^{\beta n p}} \phi\left(2^{n} x, 2^{n} y\right)^{p} \leq \phi(x, y)^{p} \lim _{n \rightarrow \infty}\left(2^{1-2 \beta} L\right)^{n p}=0
\end{aligned}
$$

for all $x, y \in \mathbb{R}$, and so, by Lemma $2.1, \mathcal{F}$ is a quadratic function. Taking $m \rightarrow \infty$ in (2.30) with $k=0$, we have

$$
\|f(x)-\mathcal{F}(x)\|_{\mathcal{Y}} \leq \frac{\widehat{\Phi}(x)}{\sqrt[p]{4^{\beta p}-(2 L)^{p}}} .
$$

Next, we assume that there exists a quadratic function $\mathcal{G}: \mathbb{R} \rightarrow \mathcal{Y}$ which satisfies the functional equation (1.3) and (2.28). Then we have

$$
\left\|\mathcal{G}(x)-\frac{1}{4^{n}} f\left(2^{n} x\right)\right\|_{\mathcal{Y}}^{p} \leq \frac{1}{4^{\beta n p}}\left\|\mathcal{G}\left(2^{n} x\right)-f\left(2^{n} x\right)\right\|_{\mathcal{Y}}^{p} \leq \frac{\widehat{\Phi}(x)^{p}}{4^{\beta p}-(2 L)^{p}}\left(2^{1-2 \beta} L\right)^{n p}
$$

for all $x \in \mathbb{R}$. Letting $n \rightarrow \infty$, we have the uniqueness of $\mathcal{F}(x)$. This completes the proof.

Theorem 2.10 Let $f: \mathbb{R} \rightarrow \mathcal{Y}$ be a $\phi$-approximatively radical quadratic function with $f(0)=0$. Assume that the function $\phi$ is expansively superadditive with a constant $L$ satisfying $2^{2 \beta-1} L<1$. Then there exists a unique quadratic function $\mathcal{F}: \mathbb{R} \rightarrow \mathcal{Y}$ which satisfies (1.3) and the inequality

$$
\|f(x)-\mathcal{F}(x)\|_{\mathcal{Y}} \leq \frac{\widehat{\Phi}(x)}{\sqrt[p]{\left(2 L^{-1}\right)^{p}-4^{\beta p}}}
$$

for all $x \in \mathbb{R}$. 
Proof From (2.29) in the proof of Theorem 2.9, we get

$$
\left\|4^{k} f\left(2^{-k} x\right)-4^{k+1} f\left(2^{-k-1} x\right)\right\|_{\mathcal{Y}} \leq 4^{\beta k} \widehat{\Phi}\left(2^{-k-1} x\right)
$$

for all $x \in \mathbb{R}$. For integers $k, m$ with $m>k \geq 0$, we get

$$
\left\|4^{k} f\left(2^{-k} x\right)-4^{m} f\left(2^{-m} x\right)\right\|_{\mathcal{Y}}^{p} \leq \frac{\widehat{\Phi}(x)^{p}}{4^{\beta p}} \sum_{j=k+1}^{m}\left(2^{2 \beta-1} L\right)^{j p}
$$

The remaining part follows as the proof of Theorem 2.9. This completes the proof.

Theorem 2.11 Let $f: \mathbb{R} \rightarrow \mathcal{Y}$ be a $\psi$-approximatively radical quadratic function with $f(0)=0$. Assume that the function $\psi$ is contractively subquadratic with a constant $L$ satisfying $2^{2-4 \beta} L<1$. Then there exists a unique quartic function $\mathcal{H}: \mathbb{R} \rightarrow \mathcal{Y}$ which satisfies (1.4) and the inequality

$$
\|f(x)-\mathcal{H}(x)\|_{\mathcal{Y}} \leq \frac{\widehat{\Psi}(x)}{2^{\beta} \sqrt[p]{16^{\beta p}-(4 L)^{p}}}
$$

where

$$
\widehat{\Psi}(x)=2 K^{3}\left(4^{\beta}\left(\psi(x, x)+\frac{1}{2} \psi(\sqrt{2} x, 0)\right)+\psi(\sqrt{2} x, \sqrt{2} x)+\frac{1}{2} \psi(2 x, 0)\right)
$$

for all $x \in \mathbb{R}$

Proof Using (2.26) in the proof of Theorem 2.7, we get

$$
\left\|f(x)-\frac{1}{16} f(2 x)\right\|_{\mathcal{Y}} \leq \frac{\widehat{\Psi}(x)}{2^{5 \beta}}
$$

for all $x \in \mathbb{R}$. Then it follows from (2.32) that

$$
\begin{aligned}
& \left\|\frac{1}{16^{k}} f\left(2^{k} x\right)-\frac{1}{16^{m}} f\left(2^{m} x\right)\right\|_{\mathcal{Y}}^{p} \\
& \leq \frac{1}{2^{5 \beta p}} \sum_{j=k}^{m-1} \frac{1}{16^{j \beta p}} \widehat{\Psi}\left(2^{j} x\right)^{p} \leq \frac{\widehat{\Psi}(x)^{p}}{2^{5 \beta p}} \sum_{j=k}^{m-1}\left(2^{2-4 \beta} L\right)^{j p}
\end{aligned}
$$

for all $x \in \mathbb{R}$ and $m>k \geq 0$. Then $\left\{\frac{1}{16^{n}} f\left(2^{n} x\right)\right\}$ is a Cauchy sequence in $\mathcal{Y}$, and it converges to a point in $\mathcal{Y}$. We can define a function $\mathcal{H}: \mathbb{R} \rightarrow \mathcal{X}$ by

$$
\mathcal{H}(x):=\lim _{n \rightarrow \infty} \frac{1}{16^{n}} f\left(2^{n} x\right)
$$

for all $x \in \mathbb{R}$. From (2.9), the following inequality holds:

$$
\begin{aligned}
& \left\|\mathcal{H}\left(\sqrt{x^{2}+y^{2}}\right)+\mathcal{H}\left(\sqrt{\left|x^{2}-y^{2}\right|}\right)-2 \mathcal{H}(x)-2 \mathcal{H}(y)\right\|_{\mathcal{Y}}^{p} \\
& \leq \psi(x, y)^{p} \lim _{n \rightarrow \infty}\left(2^{2-4 \beta} L\right)^{n p}=0
\end{aligned}
$$


for all $x, y \in \mathbb{R}$. Thus the function $\mathcal{H}$ is quartic. Taking the limit $m \rightarrow \infty$ in (2.33) with $k=0, \mathcal{H}$ satisfies (2.31) near the approximate function $f$ of the functional equation (1.4). The remaining proof is similar to that of Theorem 2.9. This completes the proof.

Theorem 2.12 Let $f: \mathbb{R} \rightarrow \mathcal{Y}$ be a $\psi$-approximatively radical quadratic function with $f(0)=0$. Assume that the function $\psi$ is expansively superquadratic with a constant $L$ satisfying $2^{4 \beta-2} L<1$. Then there exists a unique quartic function $\mathcal{H}: \mathbb{R} \rightarrow \mathcal{Y}$ which satisfies (1.4) and the inequality

$$
\|f(x)-\mathcal{H}(x)\|_{\mathcal{Y}} \leq \frac{\widehat{\Psi}(x)}{2^{\beta} \sqrt[p]{\left(4 L^{-1}\right)^{p}-16^{\beta p}}}
$$

for all $x \in \mathbb{R}$

\section{Competing interests}

The authors declare that they have no competing interests.

\section{Authors' contributions}

All authors conceived of the study, participated in its design and coordination, drafted the manuscript, participated in the sequence alignment, and approved the finial manuscript.

\section{Author details}

'Department of Mathematics, Dongeui University, Busan, 614-714, Korea. ${ }^{2}$ Department of Mathematics Education and the RINS, Gyeongsang National University, Chinju, 660-701, Korea. ${ }^{3}$ Department of Mathematics, Semnan University, P.O Box 35195-363, Semnan, Iran. ${ }^{4}$ Center of Excellence in Nonlinear Analysis and Applications (CENAA), Semnan University, Semnan, Iran.

\section{Acknowledgements}

This research was supported by Dongeui University (2011AA088) and the Basic Science Research Program through the National Research Foundation of Korea (NRF) funded by the Ministry of Education, Science and Technology (Grant Number: 2011-0021821).

Received: 1 December 2011 Accepted: 9 August 2012 Published: 31 August 2012

\section{References}

1. Ulam, SM: A Collection of Mathematical Problems. Interscience, New York (1960)

2. Ulam, SM: Problems in Modern Mathematics. Wiley, New York (1964)

3. Hyers, DH: On the stability of the linear functional equation. Proc. Natl. Acad. Sci. USA 27, $222-224$ (1941)

4. Aoki, T: On the stability of the linear transformation in Banach spaces. J. Math. Soc. Jpn. 2, 64-66 (1950)

5. Bourgin, DG: Classes of transformations and bordering transformations. Bull. Am. Math. Soc. 57, 223-237 (1951)

6. Rassias, TM (ed.): Functional Equations, Inequalities and Applications. Kluwer Academic, Dordrecht (2003)

7. Rassias, TM: On the stability of functional equations and a problem of Ulam. Acta. Appl. Math. 62, 23-130 (2000)

8. Rassias, TM: On the stability of the linear function in Banach spaces. Proc. Am. Math. Soc. 72, 297-300 (1978)

9. Rassias, TM, Šemrl, P: On the behavior of mappings which do not satisfy Hyers-Ulam stability. Proc. Am. Math. Soc 114, 989-993 (1992)

10. Rassias, JM: Solution of a problem of Ulam. J. Approx. Theory 57, 268-273 (1989)

11. Rassias, JM: On approximation of approximately linear functions by linear functions. J. Funct. Anal. 46, 126-130 (1982)

12. Forti, GL: The stability of homomorphisms and amenability, with applications to functional equations. Abh. Math. Semin. Univ. Hamb. 57, 215-226 (1987)

13. Găvruta, P: A generalization of the Hyers-Ulam-Rassias stability of approximately additive functions. J. Math. Anal. Appl. 184, 431-436 (1994)

14. Gajda, Z, Ger, R: Subadditive multifunctions and Hyers-Ulam stability. In: General Inequalities 5 (Oberwolfach, 1986), 281-291, Intern. Schriftenreihe Numer. Math., vol. 80. Birkhäuser, Basel (1987)

15. Gruber, PM: Stability of isometries. Trans. Am. Math. Soc. 245, 263-277 (1978)

16. Baktash, E, Cho, YJ, Jalili, M, Saadati, R, Vaezpour, SM: On the stability of cubic mappings and quadratic mappings in random normed spaces. J. Inequal. Appl. 2008, Article ID 902187 (2008)

17. Cho, YJ, Gordji, ME, Zolfaghari, S: Solutions and stability of generalized mixed type QC functional equations in random normed spaces. J. Inequal. Appl. 2010, Article ID 403101 (2010)

18. Cho, YJ, Kang, Jl, Saadati, R: Fixed points and stability of additive functional equations on the Banach algebras. J. Comput. Anal. Appl. 14, 1103-1111 (2012)

19. Cho, YJ, Park, C, Saadati, R: Functional inequalities in non-Archimedean in Banach spaces. Appl. Math. Lett. 23 1238-1242 (2010) 
20. Cho, YJ, Saadati, R: Lattice non-Archimedean random stability of ACQ functional equation. Adv. Differ. Equ. (2010). doi:10.1186/1687-1847-2011-31

21. Gordji, ME, Kenary, HA, Rezaei, H, Lee, YW, Kim, GH: Solution and Hyers-Ulam-Rassias stability of generalized mixed type additive-quadratic functional equations in fuzzy Banach spaces. Abst. Appl. Anal. 2012, Article ID 953938 (2012)

22. Gordji, ME, Khodaei, H, Ebadian, A, Kim, GH: Nearly radical quadratic functional equations in p-2-normed spaces. Abst. Appl. Anal. 2012, Article ID 896032 (2012)

23. Gordji, ME, Khodaei, $\mathrm{H}$ : On the generalized Hyers-Ulam-Rassias stability of quadratic functional equations. Abst. Appl. Anal. 2009, Article ID 923476 (2009)

24. Gordji, ME, Parviz, M: On the Hyers-Ulam stability of the functional equation $f\left(\sqrt{x^{2}+y^{2}}\right)=f(x)+f(y)$. Nonlinear Funct. Anal. Appl. 14, 413-420 (2009)

25. Lee, YH, Jung, SM: A fixed point approach to the stability of an $n$-dimensional mixed-type additive and quadratic functional equation. Abst. Appl. Anal. 2012, Article ID 482936 (2012)

26. Lee, YH, Jung, SM, Rassias, TM: Stability of the $n$-dimensional mixed-type additive and quadratic functional equation in non-Archimedean normed spaces. Abst. Appl. Anal. 2012, Article ID 401762 (2012)

27. Najati, A, Cho, YJ: Generalized Hyers-Ulam stability of the pexiderized Cauchy functional equation in non-Archimedean spaces. Fixed Point Theory Appl. 2011, Article ID 309026 (2011)

28. Najati, A, Kang, Jl, Cho, YJ: Local stability of the pexiderized Cauchy and Jensen's equations in fuzzy spaces. J. Inequal. Appl. 2011, 78-85 (2011). doi:10.1186/1029-242x-2011-78

29. Park, C, Cho, YJ, Kenary, HA: Orthogonal stability of a generalized quadratic functional equation in non-Archimedean spaces. J. Comput. Anal. Appl. 14, 526-535 (2012)

30. Saadati, R, Cho, YJ, Vahidi, J: The stability of the quartic functional equation in various spaces. Comput. Math. Appl. 60 1994-2002 (2010)

31. Savadkouhi, MB, Gordji, ME, Ghobadipur, N: On the Cauchy-Rassias stability of the functional equation $r^{2} f\left(\frac{s x^{2}+t y^{2}}{r}\right)=s f(x)+t f(y)$. Nonlinear Funct. Anal. Appl. 14, 665-675 (2009)

32. Lee, SH, Im, SM, Hawng, IS: Quartic functional equations. J. Math. Anal. Appl. 307, 387-394 (2005)

33. Tober, J: Stability of Cauchy functional equation in quasi-Banach spaces. Ann. Pol. Math. 83, 243-255 (2004)

34. Gordji, ME, Khodaei, H, Kim, HM: Approximate quartic and quadratic mappings in quasi-Banach spaces. Int. J. Math. Math. Sci. 2011, Article ID 734567 (2011)

35. Kang, D: On the stability of generalized quartic mappings in quasi- $\beta$-normed spaces. J. Inequal. Appl. 2010, Article ID 198098 (2010)

36. Rassias, JM, Kim, HM: Generalized Hyers-Ulam stability for general additive functional equation in quasi- $\beta$-normed spaces. J. Math. Anal. Appl. 356, 302-309 (2009)

37. Ravi, K, Rassias, JM, Kodandan, R: Generalized Ulam-Hyers stability of an AQ-functional equation in quasi-beta-normed spaces. Math. Aeterna 1, 217-236 (2011)

38. Khodaei, H, Gordji, ME, Kim, SS, Cho, YJ: Approximation of radical functional equations related to quadratic and quartic mappings. J. Math. Anal. Appl. 395, 284-297 (2012)

doi:10.1186/1029-242X-2012-186

Cite this article as: Kim et al.: On the generalized Hyers-Ulam-Rassias stability problem of radical functional equations. Journal of Inequalities and Applications 2012 2012:186.

\section{Submit your manuscript to a SpringerOpen ${ }^{\circ}$ journal and benefit from:}

- Convenient online submission

- Rigorous peer review

- Immediate publication on acceptance

- Open access: articles freely available online

- High visibility within the field

- Retaining the copyright to your article 